\title{
ENHANCING PERFORMANCE OF ITERATIVE HEURISTICS FOR VLSI NETLIST PARTITIONING
}

\author{
Sadiq M. Sait, Aiman H. El-Maleh and Raslan H. Al-Abaji Dhahran 31261, Saudi Arabia
\{sadiq,aimane,raslan\}@ccse.kfupm.edu.sa \\ King Fahd University of Petroleum and Minerals, Computer Engineering,
}

\begin{abstract}
In this paper we, present a new heuristic called PowerFM which is a modification of the well-known Fidducia Mattheyeses algorithm for VLSI netlist partitioning. PowerFM considers the minimization of power consumption due to the nets cut. The advantages of using PowerFM as an initial solution generator for other iterative algorithms, in particular Genetic Algorithm (GA) and Tabu Search (TS), for multiobjective optimization is investigated. A series of experiments are conducted on ISCAS-85/89 benchmark circuits to evaluate the efficiency of the PowerFM algorithm. Results suggest that this heuristic would provide a good starting solution for multiobjective optimization using iterative algorithms.
\end{abstract}

\section{INTRODUCTION}

In recent years, the focus of portable devices has shifted from low throughput devices (e.g., watches, calculators) to high performance devices like notebook computers, cellular phones, etc. Minimizing power is the primary concern for these battery-powered products as for such products longer battery life translates to extended use and better marketability. Exploring the tradeoffs between power, performance, and other objectives during synthesis and physical design is thus demanding more attention.

The optimization for power consumption can be performed at various levels of VLSI design including behavioral level, architectural level, logic level, and physical level. Another compelling reason for the desire of low power consumption is the increasing density of VLSI circuits. The present technology allows integration of tens of millions of transistors on a single chip and the still advancing technology is allowing further high integration. The excessive power consumption of high density circuits results in heating and thus becoming a hindrance towards high integration and hence the feasible packaging of circuits $[1,2]$. Also, circuits are operating at much higher clock frequency than before. Therefore, the power dissipation which is a function of clock frequency, is getting significantly prominent. This phenomenon is offering an obstacle in further increase of clock frequency. Due to these reasons, there is an emerging need for minimizing the power requirement of VLSI circuits. For the partitioning phase, two low-power oriented techniques based on Simulated Annealing (SA) algorithm have recently been presented in [3]. An enumerative optimal delay partitioning algorithm targeting low power is proposed by Vainshav et al. in [4].

\subsection{FM Partitioning Heuristic}

The FM heuristic is a modification of the Kernighan-Lin group migration method for circuit partitioning. In the FM algorithm, all nodes initially in the free set are arranged into a bucket array data structure, in which each bucket contains nodes with the same gain. For each move, the node with the highest gain is

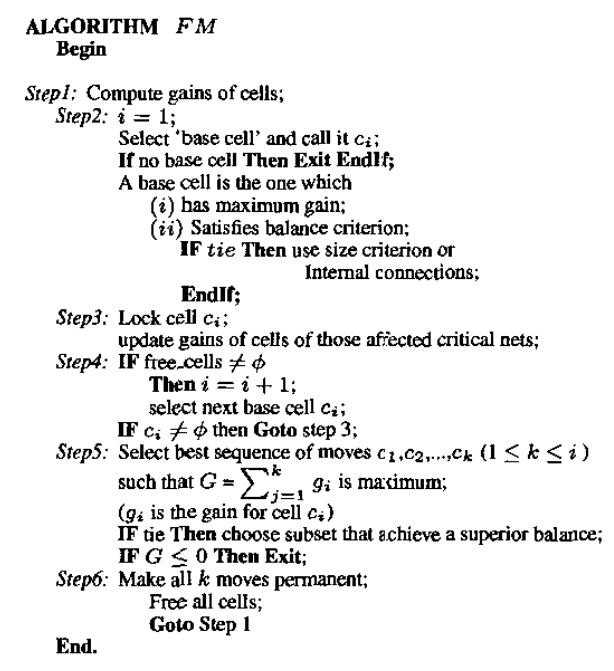

Figure 1. Fiduccia-Mattheyeses bipartitioning algorithm [5].

considered as the primary candidate to be moved from its current block (From block) to its complementary block (To block). The candidate node must satisfy the balance criterion, used to control the size of subcircuits. If the candidate node does not meet the balance criterion, the node with the next highest gain meet the balance criterion, the node with the next highest gain
is selected from the free nodes subset and moved. The moved
node is locked and eliminated from the bucket array. The move is completed by modifying the gains of all nodes connected to the critical nets. At the end of a pass, all cells are freed and the process is repeated until we reach a position where no further gain can be achieved. The best partition encountered during the pass is taken as the output of the pass. The number of cells to move is given by the value of $k$ which yields maximum positive gain $G_{k}$, where $G_{k}=\sum_{i=1}^{k} g_{i}$. Only the cells given by the best sequence, that is $c_{1}, c_{2}, \ldots, c_{k}$, ane permanently moved to their complementary blocks. Then all cells are freed and the procedure is repeated from the beginning. A general description of the heuristic is given in Fig. 1. The thest candidate node is defined according to the highest cut-gain associated with moving a node from one subcircuit to another. They are measured using the netcut model [5]. A net is called a cut net if it belongs to the current cut set; otherwise, the net is refened to as a nocut net. A net is called critical if it is a cut net that, as a result of moving a single node, can become a nocut net, or vice versa.

The basic concept of min-cut gain calculation provided with the net-cut model can be explained as follows. Let node $i_{0}$ be connected to $n$ critical cut nets and to $m$ critical nocut nets. The gain associated with the reassignrient of a node $i_{0}$ is defined as the difference:

$$
G_{i_{0}} \triangleq n-m
$$


In this work, an extension to the FM algorithm which considers optimizing power as the main objective of Partitioning is presented.

\section{PROBLEM FORMULATION AND COST FUNCTIONS}

This work addresses the problem of VLSI netlist partitioning with the objectives of optimizing power consumption, timing performance (delay), and cut-set while considering the Balance constraint (same as area constraint, as unit area is assumed for every gate). Formally, the problem can be stated as follows:

Given a set of modules $V=\left\{v_{1}, v_{2}, \ldots, v_{n}\right\}$, the purpose of partitioning is to assign the modules to a specified number of clusters $k$ (two in our case) satisfying prescribed properties. In general, a circuit can have multi-pin connections (nets) apart from two-pin. Our task is to divide $V$ into 2 subsets (blocks) $V_{0}$ and $V_{1}$ in such a way that the objectives are optimized, subject to some constraints.

Cutsize The cutsize cost function can be written as follows :

$$
\text { Minimize } f=\sum_{e \in \psi} w(e)
$$

where $\psi \subset E$ denotes the set of off-chip wires. The weight $w(e)$ on the edge $e$ represents the cost of wiring the corresponding connection as an external wire.

Delay In the general delay model where gate delay $d(v)$ and constant inter-chip wire delay are considered, $d_{c} \gg d(v)$ where $d_{c}$ is actually due to the off-chip capacitance denoted as $C_{o f f}$. Let the delay of node $v_{i} \in V$ be $d\left(v_{i}\right)$ and the delay of net $e_{k} \in E$ which is cut be $d_{c}$. Given a partition $\Phi:\left(V_{A} ; V_{B}\right)$, the path delay $\boldsymbol{d}\left(p_{i j}\right)$ between nodes $v_{i}$ and $v_{j}$ is the sum of the node delays $d\left(v_{i}\right) \in V\left(p_{i j}\right)$ and the delay of nets which are cut, that is :

$$
\text { Minimize } d\left(p_{i j}\right)=\sum_{v_{i} \in V\left(p_{i j}\right)} d\left(v_{i}\right)+d_{c} \times n c u t\left(p_{i j}\right)
$$

Power The average dynamic power consumed by a CMOS logic gate in a synchronous circuit is given by:

$$
P_{i}^{\text {average }}=0.5 \frac{V_{d d}^{2}}{T_{c y c l e}} C_{i}^{\text {load }} N_{i}
$$

where $C_{i}^{\text {load }}$ is the load capacitance, $V_{d d}$ is the supply voltage, $T_{\text {cycle }}$ is the global clock period, and $N_{i}$ is the number of gate output transitions per clock cycle. $N_{i}$ is calculated using the symbolic simulation technique of [6] under a zero delay model. $C_{i}^{\text {lood }}$ in Eq. 4 consists of two components: $C_{i}^{\text {basic }}$ which accounts for the load capacitances driven by a gate before circuit partitioning, and the extra load $C_{i}^{\text {extra }}$ which accounts for the additional load capacitance due to the external connections of the net after circuit partitioning. Then, the total power dissipation of any circuit $\zeta$ is:

$$
P_{\zeta}=\beta \frac{V_{d d}^{2}}{T_{\text {cycle }}} \sum_{i \in \zeta}\left(C_{i}^{\text {basic }}+C_{i}^{\text {extra }}\right) N_{i}
$$

where $\beta$ is a constant that depends on technology. When a circuit partitioning corresponds to a physical partitioning, $C_{i}^{\text {extra }}$ of a gate that is driving an external net is much larger than $C_{i}^{\text {basic }}$.

Area or Balance Constraint The balance constraint is given as follows:

$$
\frac{\left|\beta_{1}-\beta_{2}\right|}{\phi} \leq \alpha
$$

where $\beta_{i}$ is the number of cells in partition $i$ and $\phi$ is the total number of cells in the circuit, and the balance factor $\alpha(0.5<$ $\alpha<1.0)$.

\subsection{Overall Fuzzy Cost Function}

In order to solve the multiobjective partitioning problem, linguistic variables are defined as: cut-set, power dissipation, delay and balance. The following fuzzy rule is used to combine the conflicting objectives:

IF a solution has

Small cut-set AND

Low power consumption AND

Short delay AND

Good Balance

THEN it is a $G O O D$ solution

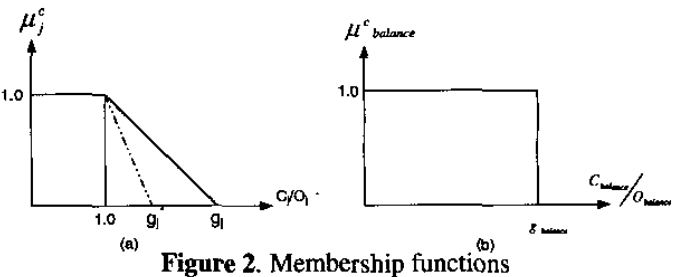

The above rule is translated to and-like OWA fuzzy operator [7] and the membership $\mu(x)$ of a solution $x$ in fuzzy set good solution is given as:

$$
\begin{array}{r}
\mu_{p d c b}^{c}(x)=\beta^{\circ} \times \min \left(\mu_{p}^{c}(x), \mu_{d}^{c}(x), \mu_{c}^{c}(x), \mu_{b}^{c}(x)\right)+ \\
\left(1-\beta^{c}\right) \times \frac{1}{4} \sum_{j=p, d, c, b} \mu_{j}^{c}(x)
\end{array}
$$

where $\mu^{\mathrm{c}}(x)$ is the membership of solution $x$ in fuzzy set of acceptable solutions, $\mu_{p d c b}^{c}(x)$ is the membership value in the fuzzy sets of " within acceptable power", "within acceptable delay", "within acceptable cut-set" and "within acceptable balance" respectively. $\beta^{c}$ is the constant in the range $[0,1]$, the superscript $c$ represents the cost. In this paper, $\mu^{c}(x)$ is used as the aggregating function. The solution that results in maximum value of $\mu^{c}(x)$ is reported as the best solution found by the search heuristic.

The membership functions for fuzzy sets Low power consumption, Short delay, Small cut-set, are shown in Fig. 2(a) We can vary the preference of an objective $j$ in the overall membership function by changing the value of $g_{j}$ which represents the relative acceptable limits for each objective whrere $g_{j} \geq 1.0$. Fig. 2(b) represents the membership functions for fuzzy set good Balance. $O_{i}$ is the estimate of lower bound on the cost of an individual $i$, and $C_{i}$ is the actual cost of $i . O_{i}$ 's are independent of iteration, therefore, these are estimated only in the beginning. Whereas, $C_{i}$ has to be calculated in every iteration for every element.

\section{POWERFM HEURISTIC}

The PowerFM is a modification of the FM algorithm which seeks minimization of the power consumption due to the cut. All concepts of the FM are maintained, the major difference is that we are calculating the gain due to the sum of the switching probabilities of the cut nets. Also some other necessary modifications are done in some parts of the Algorithm that we will discuss in what follows.

\subsection{Power Gain Calculation}

The power gain for a cell $i$ is calculated using Eqn. 8. $X_{i}$ is the set of critical cut nets. $U_{i}$ is the set of critical uncut net. 


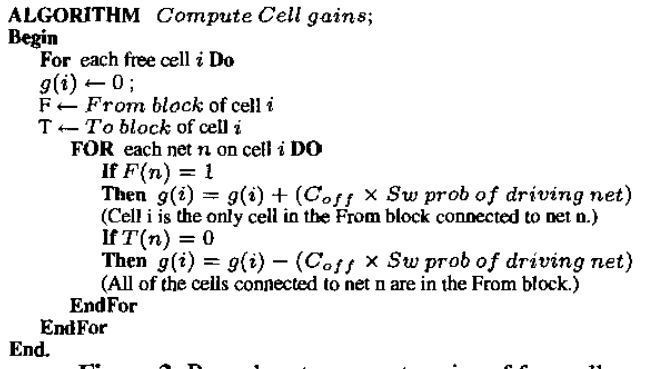

Figure 3. Procedure to compute gains of free cells.

$$
\operatorname{Pgain}(i)=C_{o f f}\left(\sum_{j \in X_{i}} S_{j}-\sum_{j \in U_{i}} S_{j}\right)
$$

In each pass, the gain of every free cell is updated according to the Compute Gain Algorithm shown in Fig. 3. Let $F(n)$ be the number of cells connected to net $n$ in the From block (current block) of the moved cell i. Let $T(n)$ be the number of cells connected to net $n$ in the To block (destination block) of the moved cell $\mathrm{i}$. When computing the gain we consider only the critical nets; A net is critical if it has a cell which if moved will change its cutsate. That is if and only if $F(n)$ is 1 , or $T(n)$ is 0 .

The algorithm is simple and it checks if the net is critical and if $F(n)=1$ then moving cell $i$ will increase the gain by $C_{o f f} \times$ Sw prob of driving net, and if $T(n)=0$ then moving the cell $i$ will decrease the gain by $C_{o f f} \times S w$ prob of driving net.

\subsection{GA and TS}

Genetic Algorithm is an elegant search technique that emulates the process of natural evolution as a means of progressing towards the optimal solution. The algorithm starts with a set of initial solutions called population that is generated randomly. In each iteration (known as generation in GA terminology), all the individual chromosomes in the population are evaluated using a fitness function. Then, in the selection step, two of the above chromosomes at a time are selected from the population. The individuals having higher fitness values are more likely to be selected. After the selection step, different operators namely crossover, mutation act on the selected individuals for evolving new individuals called offsprings.

Tabu Search starts from an initial feasible solution and carries out its search by making a sequence of random moves or perturbations. A Tabu list is maintained which stores the attributes of a number of previous moves. This list prevents taking the search process back to recently visited states. In each iteration, a subset of neighbor solutions is generated by making a certain number of moves and the best move (the move that resulted in the best solution) is accepted, provided it is not in the Tabu list. Otherwise, if the said move is in the Tabu list, it is accepted only if it leads to a solution better than the best solution found so far (aspiration criterion). Thus, the aspiration criterion can override the Tabu list restrictions. The solution encoding and initialization steps are similar to those described above for GA. These two multiobjective optimization iterative algorithms (GA and TS) for VLSI Partitioning were proposed in [8], [9], [10].

\section{EXPERIMENTAL RESULTS}

A series of experiments were performed on ISCAS-85/89 benchmark circuits, the results are analyzed and reported in this section. Table 1 shows a comparison of results of TS and GA when the initial solution is chosen frorn random or provided by Pow erFM for both GA and TS algorithms. Table 1 shows also the results obtained from PowerFM when used on its own. $P_{\text {avg }}$ refers to the average power of the results obtained from 100 runs of the PowerFM. The notation in Table1 is as follows: $D(p s)$ stands for Delay and it is measured in pico-seconds, $C u t$ is the number of nets cut, $P(s p)$ is the power dissipation measured in terms of switching probability, $T(s)$ is the total time taken by the whole run for PowerFM.

When starting from random solution it was observed that TS outperforms GA in terms of final solution costs and execution time. These two algorithms are complex, and relatively take more execution time than PowerFM. The idea of using the PowerFM as a starting solution for iterative algorithms is relevant because PowerFM proved to be an extremely fast algorithm compared to GA and TS (at least 100 times faster), with reasonable performance. This will save a lot of time for algorithms like GA and TS where the converging rate is slow. Furthermore results, showed that GA and TS were able to improve solutions provided by PowerFM. Fig. 4 and Fig. 5 shiow the performance of GA and TS respectively when applied to the circuit s1488 when starting from an initial solution provided by PowerFM. In Fig. 4 and Fig. 5, (a) shows the number of nets cut, (b) shows the longest path delay of the circuit in pico-seconds, (c) shows the power dissipation, (d) shows the cell difierence between the two partitions, (e) shows the average generation fitness, (e) shows the Best solution fitness. Both GA and TS show an improvement in terms of the overall quality of solution.
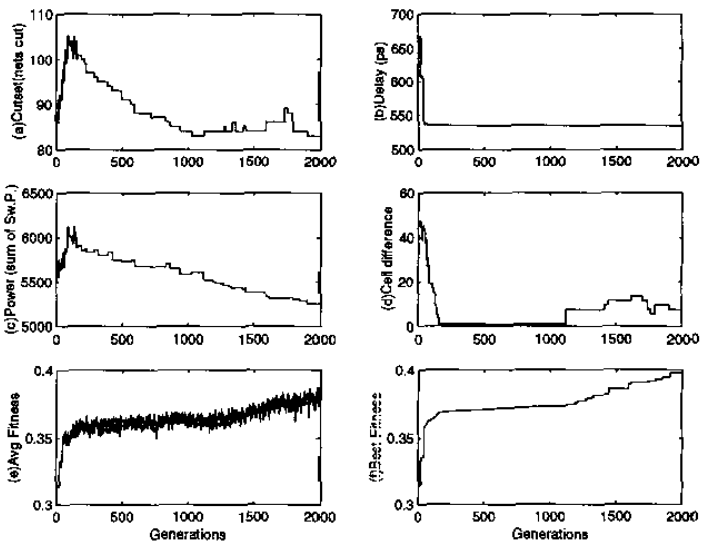

Figure 4. Genetic Algorithm starting from PowerFM for circuit s1488.

It can be noted that for most of the circuits when using a starting solution provided by PowerFM the results are better than when starting with random solution in terms of quality of solution. An important point to notice also is that although when starting from random TS performed better than GA; when starting from PowerFM GA proves to bis more efficient than TS. This is due to the fact that GA starting from a good solution has the ability to inherent the good characteristics and improve on it and proved to be able to benefit more than TS when starting from a good solution provided from PowerFM. This is noted when the results of TS (starting from PowerFM) and GA (starting from PowerFM) in Table 1 are compared; it can be seen that GA is better for large circuits ( $(3330 \ldots$... s1.5850) in terms of power and cutset. The results proved that it is beneficial to use PowerFM as a starting solution for multiobjective GA and TS. Moreover looking at the results of PowerFM alone, it comparably provided 
Table 1. Start from PowerFM versus Random Start for GA and TS.

\begin{tabular}{|c|c|c|c|c|c|c|c|c|c|c|c|c|c|c|c|c|c|}
\hline & \multicolumn{3}{|c|}{ GA Random Start } & \multicolumn{3}{|c|}{ GA Start From PowerfM } & \multicolumn{3}{|c|}{ TS Random Start } & \multicolumn{3}{|c|}{ TS Start From PowerFM } & \multicolumn{5}{|c|}{ PowerFM } \\
\hline Circuit & $\mathrm{D}(\mathrm{\rho s})$ & Cut & $\mathrm{P}(\mathrm{sp})$ & D(ps) & Cut & $P(s p)$ & D(ps) & $\mathrm{Cot}$ & $\mathrm{P}(\mathrm{sp})$ & D(ps) & Cut & $\mathrm{P}(\mathrm{sp})$ & $D(p s)$ & Cut & $\mathbf{P}(\mathrm{sp})$ & $\mathrm{T}(s)$ & $P_{\text {avg }}$ \\
\hline$\overline{S 298}$ & 233 & 19 & 1013 & $\overline{\overline{191}}$ & $\overline{\overline{10}}$ & 921 & 797 & $\overline{24}$ & $\overline{926}$ & 189 & 10 & 849 & 301 & 20 & $7 \overline{32}$ & 0.05 & $8 \overline{28}$ \\
\hline S386 & 356 & 36 & 1529 & 345 & 31 & 1401 & 386 & 30 & 1426 & 333 & 27 & 1264 & 434 & 29 & 1511 & 0.39 & 1673 \\
\hline 5641 & 1043 & 45 & 2355 & 861 & 43 & 2343 & 889 & 59 & 2281 & 844 & 48 & 2476 & 1221 & 44 & 1667 & 0.61 & 1773 \\
\hline 5832 & 444 & 45 & 3034 & 441 & 42 & 3032 & 446 & 50 & 2731 & 431 & 40 & 3135 & 527 & 51 & 2855 & 1.97 & 3338 \\
\hline S953 & 526 & 96 & 2916 & 465 & 89 & 3012 & 466 & 99 & 2518 & 430 & 85 & 2999 & 902 & 120 & 2191 & 0.60 & 2422 \\
\hline S1196 & 396 & 123 & 5443 & 390 & 86 & 4921 & 301 & 106 & 4920 & 335 & 77 & 4823 & 612 & 68 & 4116 & 1.81 & 5289 \\
\hline S1238 & 475 & 127 & 5713 & 461 & 91 & 5702 & 408 & 79 & 4597 & 401 & 74 & 5190 & 544 & 62 & 4281 & 1.80 & 5358 \\
\hline S1488 & 571 & 104 & 5648 & 541 & 83 & 5248 & 540 & 110 & 6300 & 521 & 94 & 6005 & 724 & 70 & 5228 & 5.60 & 5787 \\
\hline S1494 & 614 & 102 & 5474 & 601 & 97 & 513 & 585 & 101 & 5339 & 534 & 95 & 5058 & 630 & 80 & 5354 & 7.19 & 6022 \\
\hline S2081 & 302 & 26 & 787 & 260 & 15 & 740 & 225 & 17 & 770 & 244 & 12 & 704 & 335 & 7 & 565 & 0.11 & 586 \\
\hline 53330 & 571 & 299 & 10358 & 435 & 203 & 9296 & 533 & 295 & 10298 & 419 & 257 & 9288 & 593 & 226 & 9522 & 6.37 & 10180 \\
\hline$\$ 5378$ & 587 & 573 & 18437 & 442 & 423 & 15356 & 590 & 430 & 16527 & 432 & 400 & 15319 & 574 & 363 & 14565 & 19.22 & 15453 \\
\hline S9234 & 1313 & 1090 & 38149 & 856 & 375 & 28305 & 1052 & 918 & 34055 & 835 & 705 & 31837 & 832 & 389 & 26784 & 92.50 & 29100 \\
\hline 513207 & 1399 & 1683 & 45611 & 951 & 750 & 39620 & 843 & 1332 & 41114 & 823 & 1310 & 40235 & 1286 & 929 & 37100 & 273 & 39155 \\
\hline S15850 & 1820 & 2183 & 51747 & 1350 & 851 & 43680 & 1411 & 1671 & 47480 & 1210 & 1332 & 45320 & 1464 & 919 & 42521 & 318.56 & $4 \sqrt[3238]{ }$ \\
\hline
\end{tabular}

impressive results in terms of Power and cutset considering that its main aim is to optimize power.
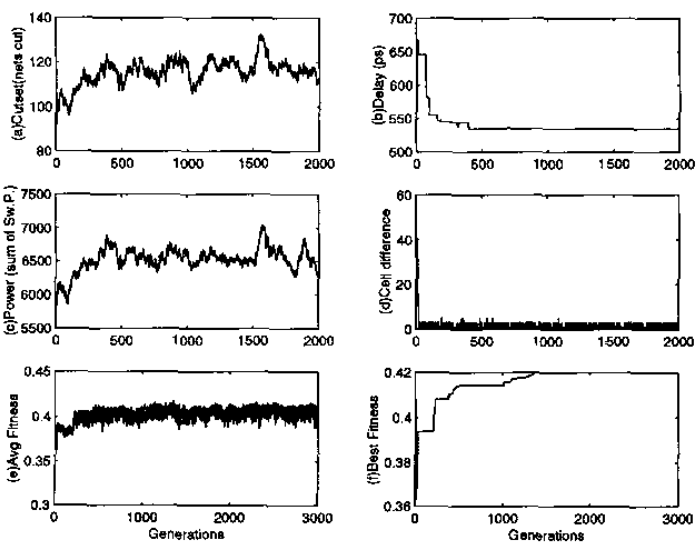

Figure 5. Tabu Search algorithm starting from PowerFM for circuit s1488.

\section{CONCLUSIONS}

In this paper, we proposed a new modification to the FM algorithm PowerFM which targets power optimization. The possibility to use the algorithm as a provider for initial solution for other iterative multiobjective algorithms in particular GA and TS was investigated. GA performed better than TS when starting from a solution rovided by PowerFM. PowerFM results where important due to its speed and good quality of the final solution. A series of experiments were performed, analyzed and reported to evaluate the efficiency of the algorithm. Results suggest that the algorithm proved to be efficient for optimizing power, and would provide a good starting solution for the multiobjective optimization using Genetic and Tabu search partitioning algorithms.

\section{Acknowledgment}

The authors thank King Fahd University of Petroleum \& Minerals, Dhahran, Saudi Arabia, for support, under project \# : COE/ITERATE/221

\section{REFERENCES}

[1] M. Pedram. CAD for Low Power: Status and Promising Directions. IEEE International Symposium on VLSI Technology, Systems and Applications, pages 331-336, 1995.
[2] U. Narayanan, G.I. Stamoulis, and R. Roy. Characterizing Individual Gate Power Sensitivity in Low Power Design. I2th International Conference on VLSI Design, pages 625 628, January 1999.

[3] I.S. Choi and S.Y. Hwang. Circuit Partitioning algorithm for Low-Power Design Under Area Constraints Using Simulated Annealing. IEE Proc. Cicrcuits Devices Systems, 146(1):8-15, February 1999.

[4] H. Vaishnav and M. Pedram. Delay optimal partitioning targeting low power VLSI circuits. IEEE Trans. on Computer Aided Design, 18(6):298-301, june 1999.

[5] C. M. Fiduccia and R. M. Mattheyses. A Linear-Time Heuristic for Improving Network Partitions. pages 175$181,1982$.

[6] A. Ghosh, S. Devadas, K. Keutzer, and J. White. Estimation of Average Switching Activity in Combinational and Sequential Circuits. Design Automation Conference, pages 253-259, 1992.

[7] R. R. Yager. On Ordered Weighted Averaging Aggregation Operators in Multicriteria Decisionmaking. IEEE Transaction on Systems, MAN, and Cybernetics, 18(1), January 1988.

[8] R. H. Al-Abaji. Evolutionary Techniques for Multiobjective VLSI Netlist Partitioning. Master's thesis, King Fahd University of Petroleum and Minerals, Dhahran, Kingdom of Saudi Arabia, May 2002.

[9] Sadiq M. Sait, Aiman El-Maleh, and Raslan Al-Abaji. Simulated evolution algorithm for multiobjective VLSI netlist bi-partitioning. Proc. of the IEEE International Symposium on Circuits and Systems (ISCAS), V:457-460, May 2003.

[10] Sadiq M. Sait, Aiman El-Maleh, and Raslan Al-Abaji. General iterative heuristics for VLSI multiobjective partitioning. Proc. of the IEEE International Symposium on Circuits and Systems (ISCAS), V:497-500, May 2003. 